\title{
Rectal hemangiopericytoma in a 37-year-old woman: a case report and review of the literature
}

\author{
Li Lu, Long Jiang Zhang, Chang Sheng Zhou and Guang Ming Lu*
}

\begin{abstract}
Introduction: Hemangiopericytoma is an uncommon perivascular tumor. Rectal Hemangiopericytomas are extremely rare. To the best of our knowledge, only two cases have been reported in the literature.
\end{abstract}

Case presentation: We report the case of a 37-year-old Asian woman with an Hemangiopericytoma rising from the anterior wall of her rectum. Abdominopelvic computed tomography showed a $7.4 \mathrm{~cm}$ solid mass between her uterus and her rectum. Heterogeneous gradual enhancement after intravenous injection of contrast material was noted with several tortuous vessels around her tumor. Intra-operative findings indicated a capsule and well-circumscribed solid tumor connecting with the anterior wall of her rectum by a small pedicle. With immunohistochemical stains, her tumor cells reacted positive for BCl-2, CD34, and ki67 and negative for CD10, CD117, S100, and Desmin. Follow-up computed tomography scans have shown no tumor recurrence or metastasis signs.

Conclusions: Rectal Hemangiopericytoma is a rare tumor with non-specific imaging findings.

Hemangiopericytomas should be included in the differential list when a massive tumor with heterogeneously gradual enhancement in the regions of the rectum is encountered.

\section{Introduction}

Hemangiopericytoma (HPC), an uncommon perivascular tumor, accounts for $1 \%$ of primary vascular tumors and occurs most frequently in the extremities, pelvis, head and neck, and meninges [1]. This tumor is generally rare in the gastrointestinal tract. Rectal HPC is extremely rare; to the best of our knowledge, only two cases have been reported in the literature in English [2]. It has been reported that some HPCs rising from the sacrum involved merely the retrorectal space [3]. Few reports on radiological findings of rectal HPCs have been published. Here, we report the clinical, ultrasonongraphy, and dynamic contrast-enhanced computed tomography (CT) findings of an HPC rising from the rectal anterior wall of a 37-year-old woman.

\section{Case presentation}

A 37-year-old Asian woman was referred to our hospital because of lower abdominal pain that began four months earlier. A vaginal palpation revealed a hard, adhering, and painless mass. Another physical examination revealed no

\footnotetext{
* Correspondence: guangminglu66@yahoo.com.cn
Department of Medical Imaging, Jinling Hospital, Nanjing, Jiangsu 210002,

* Correspondence: guangminglu66@yahoo.com.cn
Department of Medical Imaging, Jinling Hospital, Nanjing, Jiangsu 210002, China
}

(c) 2011 Lu et al; licensee BioMed Central Ltd. This is an Open Access article distributed under the terms of the Creative Commons Attribution License (http://creativecommons.org/licenses/by/2.0), which permits unrestricted use, distribution, and reproduction in any medium, provided the original work is properly cited. abnormalities. The results of laboratory tests, including complete blood count, serum electrolytes, creatinine, and urea, were normal. Our patient underwent an intra-vaginal ultrasonography (US) examination, which revealed a $6.0 \times$ $7.6 \times 6.0 \mathrm{~cm}$ solid mass between her uterus and rectum (Figure 1A). An abdominopelvic CT scan showed a $7.4 \mathrm{~cm}$ nodular solid mass between her uterus and rectum and an intense heterogeneously gradual enhancement after intravenous injection of iodinated contrast material. CT numbers of the mass ranged from 20 Hounsfield units (HU) in unenhanced CT to $70 \mathrm{HU}$ in the delayed phase (Figure 1B-E). Tortuously enhanced vessels around her tumor were also noted (Figure 1C, D). The mass encroached into the posterior part of her uterus prominently (Figure 1F) but without involving adjacent organs. No lymphoadenopathy was found. Subserosal uterine fibroid was suspected at CT. Our patient underwent tumor resection after a comprehensive evaluation of clinical and imaging findings. Intra-operative findings indicated a capsule and wellcircumscribed solid tumor connecting with the anterior wall of her rectum by a small pedicle. The gross specimen showed a well-encapsulated mass that was $10.0 \times 8.0 \times$ $5.0 \mathrm{~cm}$ in size. The external surface was pink and whitish. Microscopically, the specimen showed the features of a 


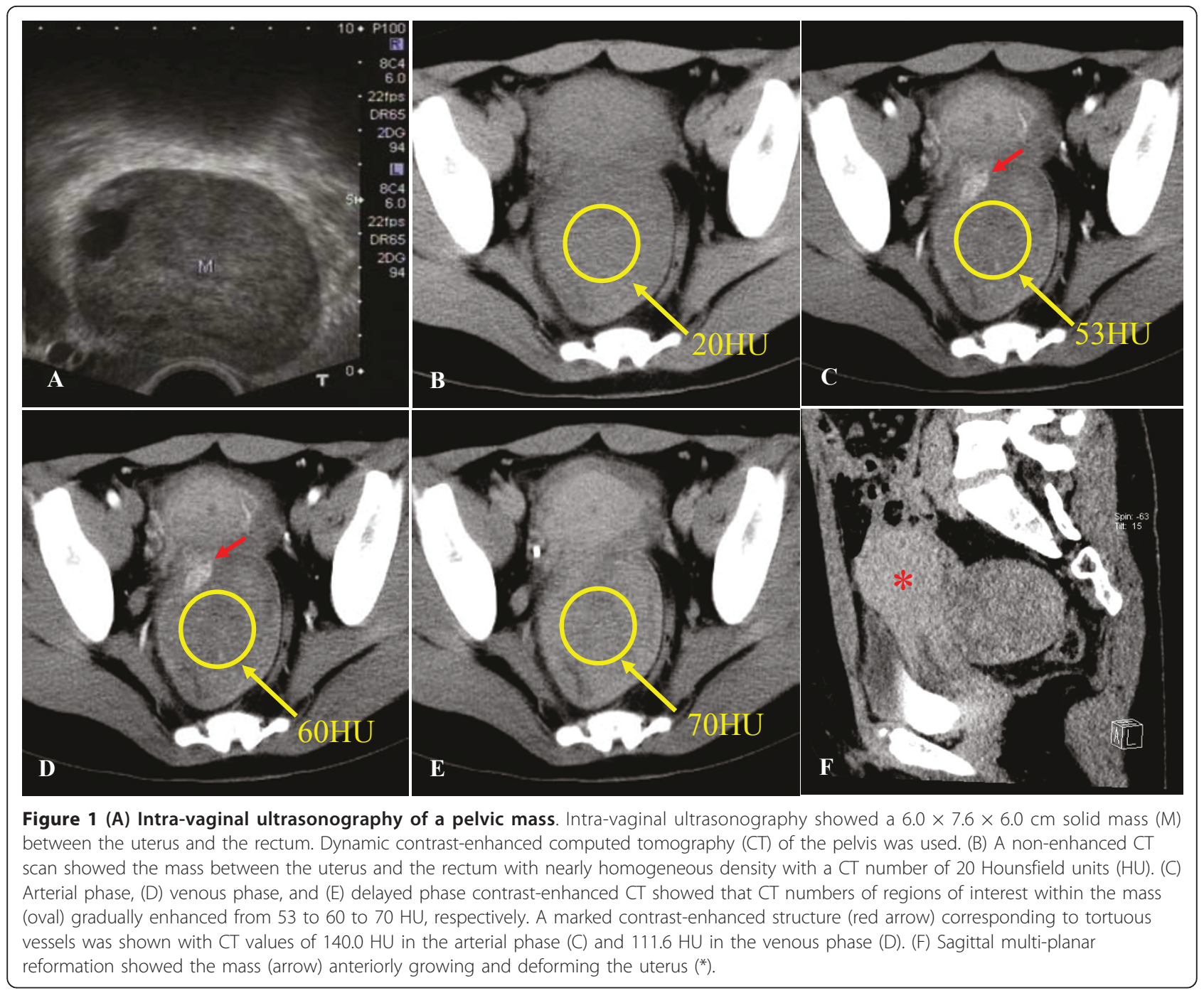

mesenchymal tumor with spindle and oval cells (Figure 2A). Branch-like blood vessels were visible within the tumors (Figure 2B). With immunohistochemical stains, tumor cells reacted positive for CD34 (Figure 2C), Bcl-2 (Figure 2D), and ki67 and negative for CD10, CD117, S100, and Desmin. The tumor had low malignant potential activity. Follow-up pelvis US and CT examinations revealed no tumor recurrence or metastasis signs six months after surgery.

\section{Discussion}

HPC was first described in 1942, by Stout and Murray [4], and has been further understood since the development of electron microscopy, immunohistochemistry, and cytogenetics in the 1970s. HPC is classified as a soft-tissue vascular tumor arising from pericytes, which are contractile cells surrounding the capillaries and post-capillary venules [5]. Consequently, HPC may occur anywhere capillaries are found. Rectal HPC is very rare; to the best of our knowledge, only two cases rising from the rectum have been described in the literature [2]. The tumor can present in patients of any age but does so predominantly in the fourth and fifth decades and has a male-to-female ratio of 1.8.

HPCs have some characteristic clinical features. One of these features is the rate of recurrence, which is as high as $52 \%$ of cases [6] (mostly in the lungs, liver, and regional lymph nodes) and which necessitates long-term follow-up after resection of the primary tumor. Other interesting features are the various para-neoplastic symptoms, including hypoglycemia [7] and hypertension [8], which accompany this neoplasm because the tumor can secrete insulin-like substances and hyper-utilize glucose. A review of the literature revealed that the size of a tumor causing hypoglycemic symptoms ranged from 12 to $27 \mathrm{~cm}$. In our patient, the size of the primary tumor was $10.0 \times 8.0 \times 5.0 \mathrm{~cm}$.

The radiographic features of rectal HPCs are nonspecific. A large HPC usually has a marked mass effect 

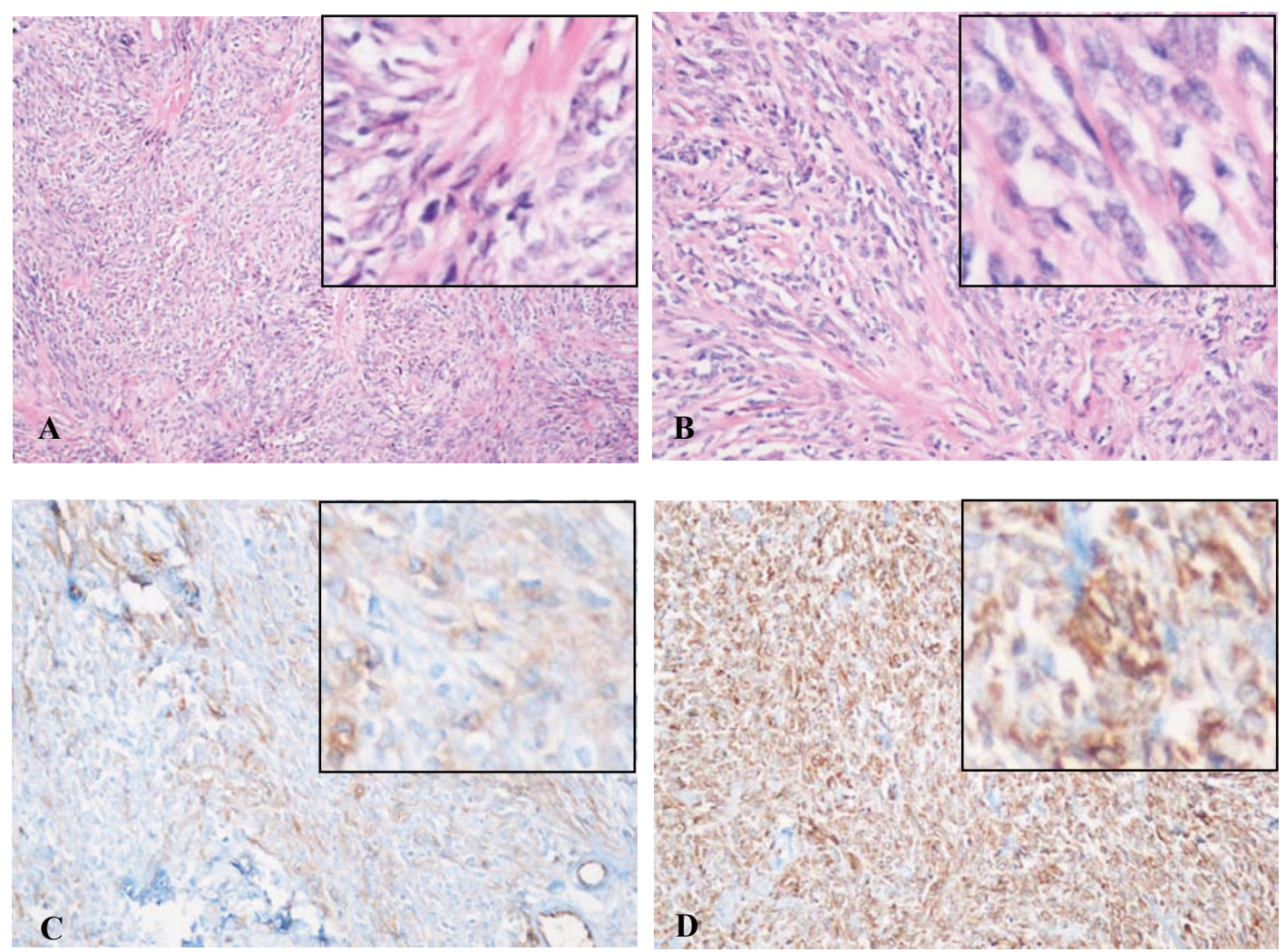

Figure 2 Histopathological images. (A) Collagen denaturation can be seen in a partial mesenchyme. Branch-like blood vessels are visible (hematoxylin and eosin $[\mathrm{H}-\mathrm{E}]$ stain, original magnification $\times 100$ ). (B) Spindle and oval tumor cells and stromal sinusoid can be observed $(\mathrm{H}-\mathrm{E}$ stain, original magnification $\times 200$ ). (C) In CD34 immunohistochemical stains, tumor cells reacted positive for CD34. (D) In Bcl-2

immunohistochemical stains, tumor cells reacted strongly positive for Bcl-2.

with necrosis and cystic changes. Calcification is rare. Intense heterogeneous gradual enhancement can be observed after intravenous injection of contrast material with several tortuous enhanced vessels around the tumor, which indicate the vascular origin of the tumor. The uncertainty of the rectal origin reflects the large exophytic nature of the tumor and its relatively small pedicle [9]. Magnetic resonance imaging (MRI) is usually chosen as the method for detecting the organ of origin of a pelvic mass. However, MRI was not performed in our patient. On MRI, HPC typically shows an intermediate signal intensity on T1-weighted images and hyper-intense serpentine channels on gadoliniumenhanced images. MRI shows a characteristic sign"flow void phenomena"-that often emerges from hypervascular tumors. Lipomatous HPCs are benign variants of HPCs [10].

Rectal HPCs need to be differentiated from three types of tumors: uterine myomas, exogenous gastrointestinal stromal tumors (GISTs) of the rectum, and retroperitoneal tumors. On MRI, non-degenerating uterine myomas show entirely or predominantly low signal intensity on T2-weighted images, and it displays differentiation between uterine myomas and HPCs because HPCs appear as high signal intensity on T2-weighted images. But degenerated uterine myomas may have varied appearances on $\mathrm{T} 2$-weighted and contrast-enhanced images according to the hyaline or myxoid degeneration, degree of interstitial edema, cystic degeneration, necrosis, fibrosis, calcification, hemorrhage, carneous degeneration, and fat.

Small tumors typically appear as homogeneous soft-tissue masses with moderate contrast enhancement, whereas large tumors often appear to have a heterogeneous density or signal intensity because of ulceration, necrosis, or cavitation. Thus, precise differential diagnosis is very difficult, but GISTs rarely cause lymph node metastasis; if extensive lymph node metastases are found, other diseases should be considered [11].

It is very difficult to differentiate retroperitoneal tumors, such as leiomyosarcoma, liposarcoma, neurogenic tumors, and malignant fibrous histiocytoma $(\mathrm{MFH})$, on the basis of imaging findings. Of these tumors, liposarcoma is one of the most common primary neoplasms in the retropenitoneum. The lipoma-like component may lead to a diagnosis of liposarcoma, although 
abdominal tumors with fat are not always liposarcomas [12]. Neurogenic tumors often occur in lateral walls of the pelvis with moderate or marked enhancement. MFH and leiomyosarcoma have non-specific imaging findings, which do not facilitate a definitive diagnosis; however, a low-signal-intensity septum of the tumor in T2-weighted images may indicate a diagnosis of MFH [13].

Tumor resection is the mainstay method of treatment of HPCs. Post-operative adjuvant radiotherapy should be offered to all patients, regardless of the degree of resection. The optimal management of local recurrence is indicated by the size of the recurrence and the overall systemic disease burden present at the time of recurrence. Post-operative radiation therapy does not confer any significant protection against the development of distant metastases. For this reason, long-term clinical and radiographic follow-up of these patients is imperative given that recurrence or metastasis or both often take several years to develop.

\section{Conclusions}

Rectal HPC is a rare tumor with non-specific imaging findings. HPCs should be included in the differential list when a massive tumor with heterogeneously gradual enhancement in the regions of the rectum is encountered.

\section{Consent}

Written informed consent was obtained from the patient for publication of this case report and any accompanying images. A copy of the written consent is available for review by the Editor-in-Chief of this journal.

\begin{abstract}
Abbreviations
CT: computed tomography; GIST: gastrointestinal stromal tumor; HPC: hemangiopericytoma; HU: Hounsfield units; MFH: malignant fibrous histiocytoma; MRI: magnetic resonance imaging; US: ultrasonography.
\end{abstract}

\section{Authors' contributions}

$\mathrm{LL}$ gathered the data, performed the literature review, and edited the manuscript. LJZ and CSZ participated in the acquisition and analysis of the literature data and helped to draft the manuscript. GML revised the final manuscript. All authors read and approved the final manuscript.

\section{Competing interests}

The authors declare that they have no competing interests.

Received: 19 October 2010 Accepted: 5 August 2011

Published: 5 August 2011

\section{References}

1. Enzinger FM, Smith BH: Hemangiopericytoma: an analysis of 106 cases. Hum Pathol 1976, 7:61-82.

2. Bacon HE, Sherman LF, Campbell WN: Hemangiopericytoma, an unusual extra rectal tumor. Minn Med 1950, 33:683-684.

3. Zentar A, Sall I, Ali AA, Bouchentouf SM, Quamous M, Chahdi H, Hajjouji A, Fahssi M, El Kaoui H, Al Bouzidi A, Marjani M, Sair K, Bousselmame N: Sacral hemangiopericytoma involving the retrorectal space: report of a case. Surg Today 2009, 39:344-348.

4. Stout AP, Murray MR: Hemangiopericytoma: a vascular tumor featuring Zimmermann's pericytes. Ann Surg 1942, 116:26-33.
5. Horikawa-Kyo Y, Tanaka T, Tanano H, Kitayama Y, Karakawa S, Taniyama K: Mediastinal hemangiopericytoma. Pediatr Blood Cancer 2009, 53:206-207.

6. Noda N, Hasegawa H, Kanai M, Shimizu Y, Yoshida H, Matsumoto T, Akita M: Recurrent hemangiopericytoma of the liver: report of a case. Surg Today 1995, 25:72-75.

7. Cotterill AM, Holly JM, Davies SC, Coulson VJ, Price PA, Wass JA: The insulin-like growth factors and their binding proteins in a case of nonislet-cell tumour-associated hypoglycaemia. J Endocrinol 1991, 131:303-311.

8. Robertson PW, Klindjian A, Harding LK, Walters G, Lee MR, Robb-Smith AH: Hypertension due to renin-secreting renal tumor. Am J Med 1967, 43:963-976.

9. Hama Y, Okizuka H, Odajima K, Hayakawa M, Kusano S: Gastrointestinal stromal tumor of the rectum. Eur Radiol 2001, 11:216-219.

10. Dozois EJ, Malireddy KK, Bower TC, Stanson AW, Sim FH: Management of a retrorectal lipomatous hemangiopericytoma by preoperative vascular embolization and a multidisciplinary surgical team: report of a case. Dis Colon Rectum 2009, 52:1017-1020.

11. Rimondini A, Belgrano M, Favretto G, Spivach A, Sartori A, Zanconati F, Cova MA: Contribution of CT to treatment planning in patients with GIST. Radiol Med 2007, 112:691-702.

12. Dooms GC, Hnicak H, Sollitto RA, Higgins CB: Lipomatous tumors and tumors with fatty component: MR imaging potential and comparison of MR and CT results. Radiology 1985, 157:479-483.

13. Agaimy A, Gaumann A, Schroeder J, Dietmaier W, Hartmann A Hofstaedter $F$, Wünsch PH, Mentzel T: Primary and metastatic high-grade pleomorphic sarcoma/malignant fibrous histiocytoma of the gastrointestinal tract: an approach to the differential diagnosis in a series of five cases with emphasis on myofibroblastic differentiation. Virchows Arch 2007, 451:949-957.

doi:10.1186/1752-1947-5-352

Cite this article as: Lu et al:: Rectal hemangiopericytoma in a 37-yearold woman: a case report and review of the literature. Journal of Medical Case Reports 2011 5:352.

\section{Submit your next manuscript to BioMed Central and take full advantage of:}

- Convenient online submission

- Thorough peer review

- No space constraints or color figure charges

- Immediate publication on acceptance

- Inclusion in PubMed, CAS, Scopus and Google Scholar

- Research which is freely available for redistribution 